\title{
乌s \\ Segmented beryllium target for a 2 MW super beam facility
}

\author{
T. Davenne, O. Caretta, C. Densham, M. Fitton, and P. Loveridge \\ STFC Rutherford Appleton Laboratory, Didcot OX11 OQX, United Kingdom \\ P. Hurh, R. Zwaska, J. Hylen, and V. Papadimitriou \\ Fermilab, Kirk Road and Pine Street, Batavia, Illinois 60510, USA
}

(Received 12 January 2015; published 14 September 2015)

\begin{abstract}
The Long Baseline Neutrino Facility (LBNF, formerly the Long Baseline Neutrino Experiment) is under design as a next generation neutrino oscillation experiment, with primary objectives to search for $C P$ violation in the leptonic sector, to determine the neutrino mass hierarchy and to provide a precise measurement of $\theta_{23}$. The facility will generate a neutrino beam at Fermilab by the interaction of a proton beam with a target material. At the ultimate anticipated proton beam power of $2.3 \mathrm{MW}$ the target material must dissipate a heat load of between 10 and $25 \mathrm{~kW}$ depending on the target size. This paper presents a target concept based on an array of spheres and compares it to a cylindrical monolithic target such as that which currently operates at the T2K facility. Simulation results show that the proposed technology offers efficient cooling and lower stresses whilst delivering a neutrino production comparable with that of a conventional solid cylindrical target.
\end{abstract}

DOI: 10.1103/PhysRevSTAB.18.091003

PACS numbers: 14.60.St, 13.85.-t

\section{INTRODUCTION}

The LBNF at FermiLab [1] is a leading candidate to become a neutrino super beam, a so-called conventional neutrino beam with a proton driver exceeding $1 \mathrm{MW}$ beam power. The proton beam interacts with a target material located inside the bore of a magnetic horn [2,3], which focuses one sign of the resulting charged pions in a forward direction. A wideband neutrino beam is generated from the decay of these pions, which is directed to a far detector some $1300 \mathrm{~km}$ distant. The Tokai to Kamiokande (T2K) experiment is an example of such a facility which is designed for a $750 \mathrm{~kW}$ beam [4]. This has been operational since 2009 and employs a helium cooled graphite cylinder as the target. The study presented here demonstrates the target challenges for a higher power super beam facility such as LBNF. Table I shows the beam parameters used for the study.

The energy deposited in the target depends on the specific beam parameters and target material. Graphite is a candidate target material due to its high temperature properties and resilience to radiation damage, and has been used in other conventional neutrino facilities [4-6]. Beryllium was selected for this study as a comparative material because of its similarly low atomic number (thus low energy deposition [7]), mechanical properties and successful application for beam windows [6]. Concerns

Published by the American Physical Society under the terms of the Creative Commons Attribution 3.0 License. Further distribution of this work must maintain attribution to the author(s) and the published article's title, journal citation, and DOI. about radiation damage in graphite also motivated our interest to investigate if beryllium is viable from a thermomechanical point of view. The relative radiation damage of beryllium compared to graphite is an active topic of research; however, it is outside the scope of this paper to try to predict target lifetime as a function of radiation damage.

We first report results from thermomechanical simulations of one meter long beryllium targets with radii equivalent to 3 times the beam $\mathrm{rms}$ dimension (or $\sigma$, ranging from 4.5 to $10.5 \mathrm{~mm}$ as reported in Table I). FLUKA [8] was used to calculate the energy deposition in the beryllium as a function of the beam parameters and to compare the useful pion yield of the various design options. Table II shows a summary of the FLUKA results indicating how the total thermal power deposited in the beryllium target increases for larger radii, but how the peak energy density is maximum for the smallest target which has the smallest beam. The ANSYS simulation packages were then applied to predict operational temperatures and stresses using the FLUKA data as an input.

\section{DISCUSSION}

A peripherally cooled cylindrical target has been successfully employed at $\mathrm{T} 2 \mathrm{~K}$ and was considered as the baseline design for this study. However given the short beam pulse and the associated rapid heating, dynamic inertial stresses are predicted to develop in the target. In the case of a beryllium cylinder at $2.3 \mathrm{MW}$ the predicted maximum stress including thermal and inertial components exceeds the practical design limit (see Fig. 1). 
TABLE I. Beam parameters at ultimate planned intensity.

\begin{tabular}{|c|c|c|c|c|c|}
\hline $\begin{array}{l}\text { Proton beam } \\
\text { energy }[\mathrm{GeV}]\end{array}$ & $\begin{array}{l}\text { Protons per } \\
\text { pulse }\end{array}$ & $\begin{array}{l}\text { Repetition } \\
\text { period [s] }\end{array}$ & $\begin{array}{l}\text { Proton beam } \\
\text { power }[\mathrm{MW}]\end{array}$ & $\begin{array}{c}\text { Beam } \sigma, \\
{[\mathrm{mm}]}\end{array}$ & $\begin{array}{c}\text { Target } \\
\text { radius }[\mathrm{mm}]\end{array}$ \\
\hline 120 & $1.6 \times 10^{14}$ & 1.33 & 2.3 & $1.5-3.5$ & $4.5-10.5$ \\
\hline Bunch length (ns) & Bunch spacing (ns) & Bunches per pulse & Protons per bunch & Pulse length $(\mu \mathrm{s})$ & \\
\hline $2-5$ & 18.8 & 519 & $3.1 \times 10^{11}$ & 9.78 & \\
\hline
\end{tabular}

The highest stress levels are expected to occur in the case of an off-center beam which although undesirable represents a plausible scenario. In addition to the stress an offcenter beam would cause significant deflection of a cylindrical target. Figure 1 reports the maximum Von Mises stress (including both the thermal stress and inertial dynamic component), in a cylindrical beryllium target as a function of how far the beam is off center. Note that the worst case scenario occurs with a beam off center of $2 \sigma$ (i.e., $3 \mathrm{~mm}$ in the case of a $9 \mathrm{~mm}$ diameter target). Also note that the stress is higher in the smaller diameter target i.e., $9 \mathrm{~mm}$ compared to $21 \mathrm{~mm}$. This is because the $9 \mathrm{~mm}$ target has a correspondingly smaller beam giving rise to a higher temperature jump per pulse and a steeper temperature gradient across the radius of the target.

An effective way to reduce the dynamic stress components in a cylindrical target is to segment the target into smaller pieces so that the ratio,

$$
\frac{L}{t c},
$$

becomes smaller than one (where $L$ is the characteristic dimension of the target, $t$ is the energy deposition time and $c$ the sound speed in beryllium). The stress in the target is also shape dependent and tends to concentrate at corners, so a sphere is an ideal segment shape.

Figure 2 shows a comparison of the evolution of the peak stress in a beryllium cylinder and in a sphere as a function of time. The stress in the cylinder is dominated by the propagation of elastic longitudinal waves traveling the entire length of the target and reflecting at the extremities. The largest amplitude oscillation period corresponds to $2 L / c$. On the other hand the dynamic component in a sphere (of equal diameter to the cylinder) is almost negligible. There is some inertial component within the

TABLE II. Results from Monte-Carlo FLUKA simulations showing range of energy deposition in a beryllium target.

\begin{tabular}{lccccc}
\hline \hline $\begin{array}{l}\text { Proton } \\
\text { beam } \\
\text { power } \\
(\mathrm{MW})\end{array}$ & $\begin{array}{c}\text { Target } \\
\text { cylinder } \\
\text { diameter } \\
(\mathrm{mm})\end{array}$ & $\begin{array}{c}\text { Beam } \sigma \\
(\mathrm{mm})\end{array}$ & $\begin{array}{c}\text { Deposited } \\
\text { energy } \\
(\mathrm{kJ} / \text { pulse })\end{array}$ & $\begin{array}{c}\text { Time } \\
\text { averaged } \\
\text { power } \\
(\mathrm{kW})\end{array}$ & $\begin{array}{c}\text { Peak ensity } \\
(\mathrm{kJ} / \mathrm{cc} / \mathrm{pulse})\end{array}$ \\
\hline 2.3 & 9 & 1.5 & 14 & 10.5 & 846 \\
2.3 & 21 & 3.5 & 30.7 & 23.1 & 245 \\
\hline \hline
\end{tabular}

first 25 microseconds but for a $17 \mathrm{~mm}$ diameter beryllium sphere the ratio above equals 0.14 and as such a large inertial component is not expected.

Figures 3 and 4 describe the layout proposed for a helium cooled segmented beryllium target. At this point it should be noted that a helium cooled stationary granular target was proposed as a target for a future neutrino factory where the target would be subjected to extremely high power density [9]. In this arrangement an array of target spheres is constrained centrally within a tube by a set of helical tubular spacers. This produces a spiralling path for helium cooling around the periphery of the spheres. Figure 3 shows a prototype with a helix and outer tube made of glass so as to allow induction heating tests of the target spheres to be carried out. The outline design as shown in Fig. 4 shows how the concept could fit within a magnetic horn employing an annular feed path for the coolant similar to that employed for a cylindrical target. It is envisaged that the outer cans and helix which are not directly in the beam would be made from a titanium alloy. Titanium alloys offer good strength and elasticity. The fit between the spheres and the helix would be designed such that the spheres are held in place, the thermal expansion of the spheres would be less than 30 microns on diameter and is not expected to

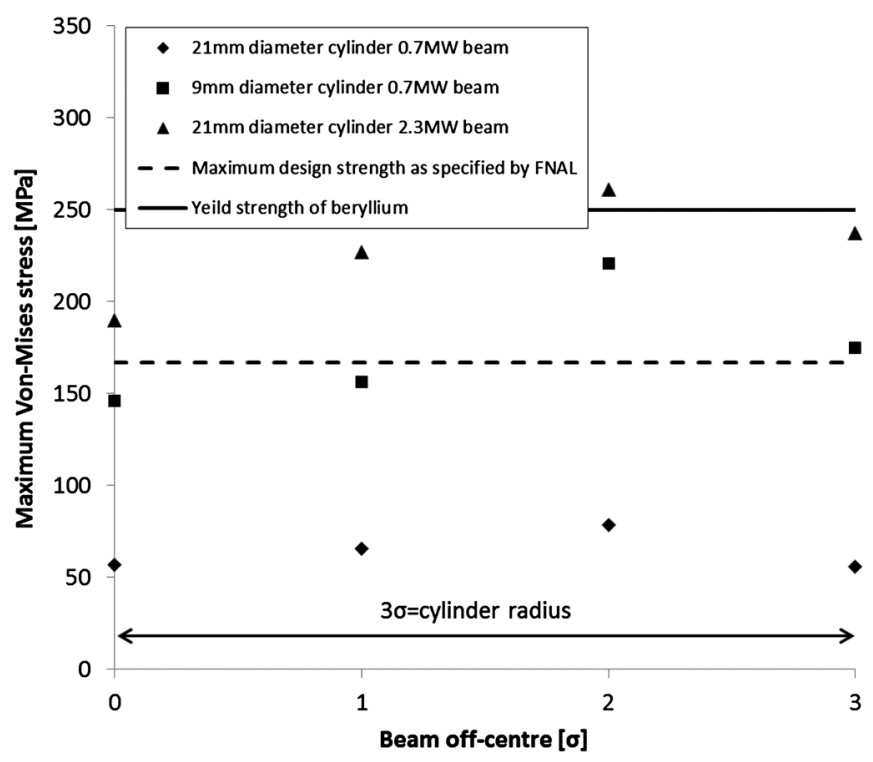

FIG. 1. Maximum Von Mises stress (including thermal and inertial components) in beryllium cylinders as a function of level of beam misalignment. 


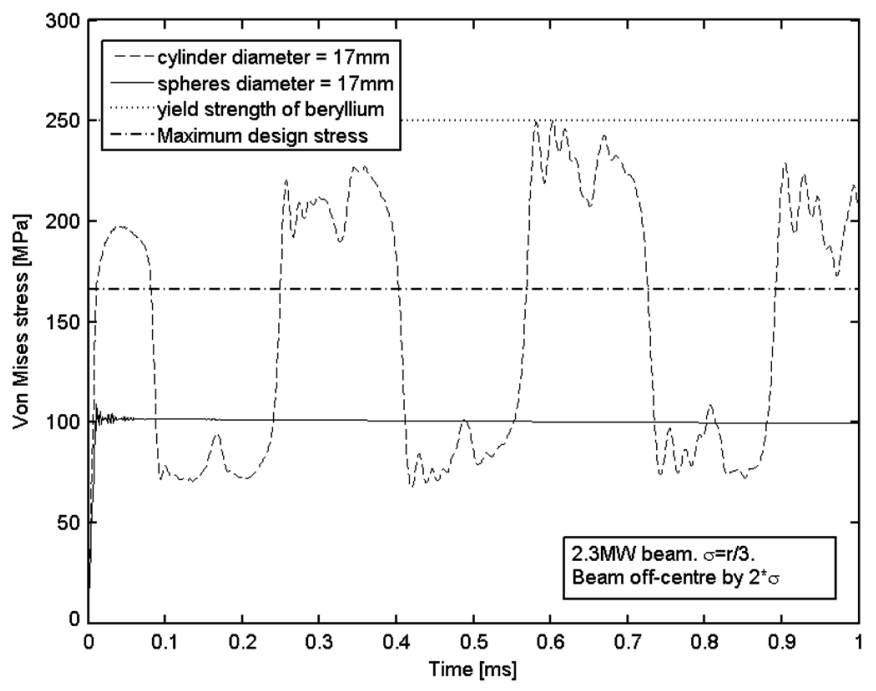

FIG. 2. Maximum Von Mises stress (including thermal and inertial components) in a beryllium cylinder and sphere both of the same diameter.

significantly stress the titanium helix which has an inherently flexible geometry. The helix would be a close fit inside the can such that it is well enough located to give good position accuracy relative to the beam but with a small amount of expansion room to avoid overstressing the inner can. The beam windows are envisaged to be either titanium or beryllium. The coolant enters the target and passes down the annular section on the outside before turning and passing through the target section. The flow is in this direction to keep the temperature of the outer surfaces of

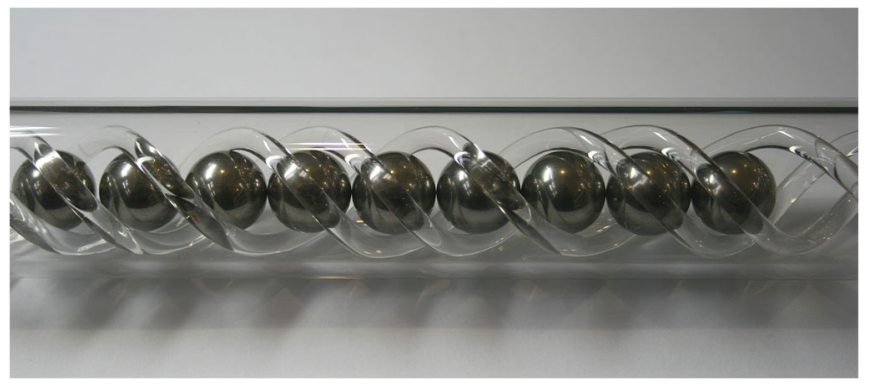

FIG. 3. Spherical array target for heat transfer tests. the target to a minimum. As such the outer can of the target should be maintained at a temperature near the helium inlet temperature, ensuring that thermal radiation from the target to the magnetic horn is kept to a minimum.

Consideration of a figure of merit defined below suggests that the optimum target radius, $r_{\text {target }}$, is approximately 3 times the beam $\sigma$, over a realistic range of diameters. Therefore target cylinders of varying radii have been investigated, keeping to the ratio:

$$
\frac{r_{\text {target }}}{\sigma}=3
$$

Both beam powers of $700 \mathrm{~kW}$ (envisaged phase 1 beam power) and 2.3 MW (ultimate anticipated beam power) have been used as inputs to the stress calculations.

Figure 5 shows the peak Von Mises stress (including thermal and inertial components) for simply supported beryllium cylinders and an array of spheres with a $2 \sigma$ offcenter beam across the range of radii as defined in Table I.

Also shown in the figure is a maximum design stress assumed as two-thirds of the nominal yield strength. As can be seen in Fig. 5, at 2.3 MW only a segmented beryllium target could survive an off-center focused beam without yielding. If the beryllium cylinder was cantilevered from one end then a significant end deflection is predicted following application of an off-center beam. Figure 6 shows the predicted transient end deflection of a cantilevered $21 \mathrm{~mm}$ diameter beryllium target resulting from a pulse from the $2.3 \mathrm{MW}$ beam hitting the target at a distance $2 \sigma$ from the center of the target.

A segmented sphere target would not see these large deflections. Note also that in normal operation as well as off-center conditions a segmented target would experience lower stresses than a cylinder hence offering a longer life expectancy.

The pion yield of the cylindrical and spherical array concepts were compared using a figure of merit (FOM). The FOM was calculated using FLUKA to obtain the yield of pions emerging from the target surface. The specific output taken from FLUKA was the plain double differential yield of pions of both signs with respect to the kinetic energy and the transverse momentum of the pions.

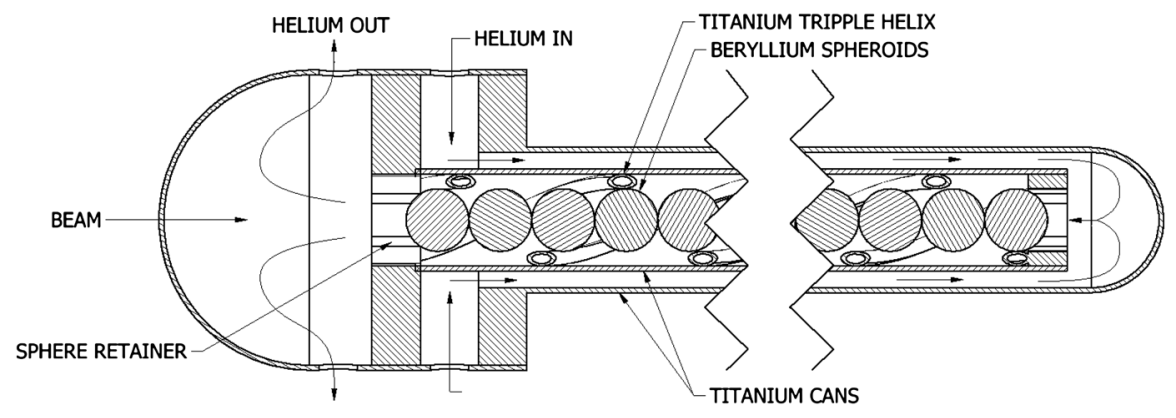

FIG. 4. Schematic diagram of proposed super beam spherical array target. 


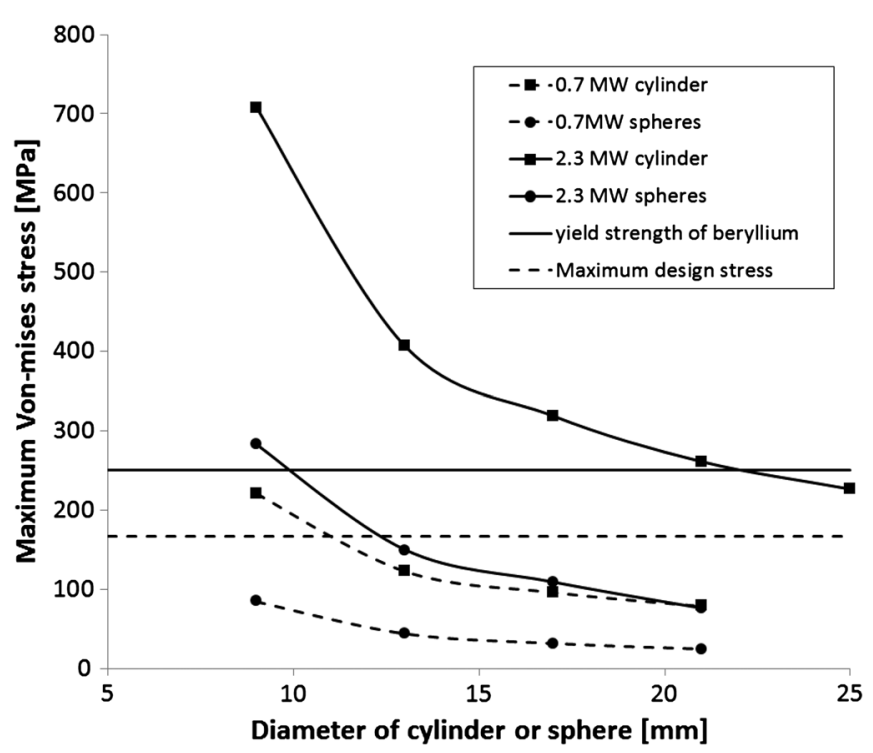

FIG. 5. Maximum Von Mises stress (including thermal and inertial components) across studied parameter space with a $2 \sigma$ off-center beam (worst case design point as seen in Fig. 1).

The FOM was then calculated as the summation of the yield in each energy interval multiplied by the corresponding weighting factor for that interval expressed mathematically as follows:

$$
\mathrm{FOM}=\sum_{n=1}^{21}\left(\operatorname{Ecen}_{n}\right)^{2.5} \int_{E_{\text {min }_{n}}}^{E_{\max _{n}}} \int_{0}^{\Delta p} \frac{\partial^{2} N}{\partial E \partial p} \partial p \partial E .
$$

In the equation, $E$ is the kinetic energy of the pions leaving the target (with transverse momentum $p$ having an acceptance of $0.4 \mathrm{GeV} / c$ ) and is scored in 21 intervals between 1.5 and $12 \mathrm{GeV}$. N is the number of pions of both signs emerging from the target per primary particle. The energy at the center of each interval was calculated as

$$
\text { Ecen }=\left(\frac{E_{\min }+E_{\max }}{2}\right) .
$$

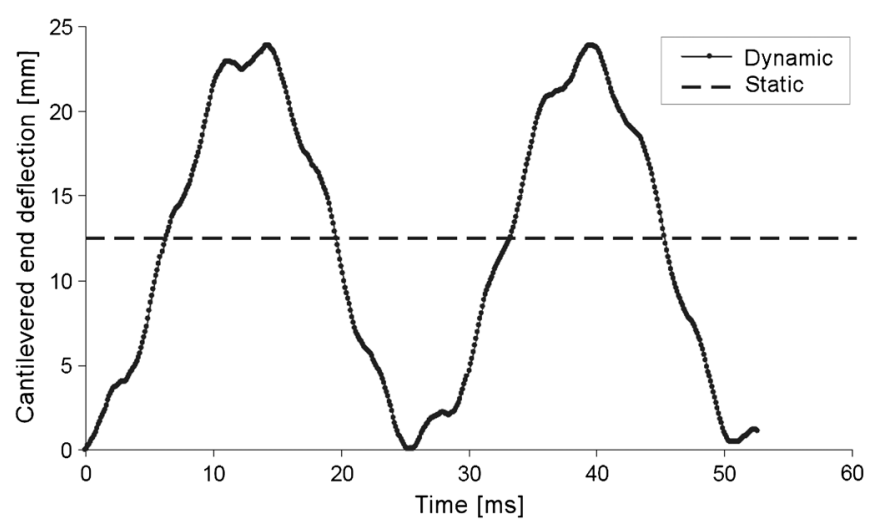

FIG. 6. End deflection of a $1 \mathrm{~m}$ long $21 \mathrm{~mm}$ diameter cantilevered beryllium cylinder with $2.3 \mathrm{MW}$ beam, applied with an offset of $2 \sigma / 7 \mathrm{~mm}$ beam offset.
The FOM was developed to allow generic optimization of target parameters without being overly specific to a particular facility. Clearly neutrino production is the true goal, and the FOM does not simulate this however it does give weight to pions in the appropriate energy range and the transverse momentum cut acts to account for the limited efficiency of the horn to focus pions with a large transverse momentum. The FOM has allowed a more rapid iteration of the target design process than would be possible if a complete facility simulation had to be performed for every target design tweak. Figure 7 shows how the FOM varies with the radius and length of the target cylinder. It points to the fact that optimum physics performance is expected with smaller radii and increased length.

Figure 8 highlights that the FOM is comparable between spheres and a cylindrical target of the same radius.

Steady state computational fluid dynamics (CFD) modeling indicates that an array of spheres could be effectively cooled with helium at the $2.3 \mathrm{MW}$ beam power. The CFD model treats the helium as an ideal gas such that changes in density due to pressure and temperature are accounted for. Temperature dependent material properties are also applied to the beryllium. A two-equation turbulence model is used to model the turbulent boundary and resulting heat transfer between the helium and the target spheres. An example sphere diameter of $13 \mathrm{~mm}$ was selected as it was the smallest size with a predicted stress below the maximum design stress (Fig. 5). Choosing the smallest possible sphere size reduces the total heat load that must be removed and is also predicted to yield better physics performance. At $13 \mathrm{~mm}$ diameter the integrated heat load on the array of spheres is $10.5 \mathrm{~kW}$. A mass flow of $24 \mathrm{~g} / \mathrm{s}$ of helium at a pressure of 10 bar gives rise to a temperature rise in the helium of $83{ }^{\circ} \mathrm{C}$ and a maximum helium velocity of $210 \mathrm{~m} / \mathrm{s}$ corresponding to a Mach number of 0.18 . The

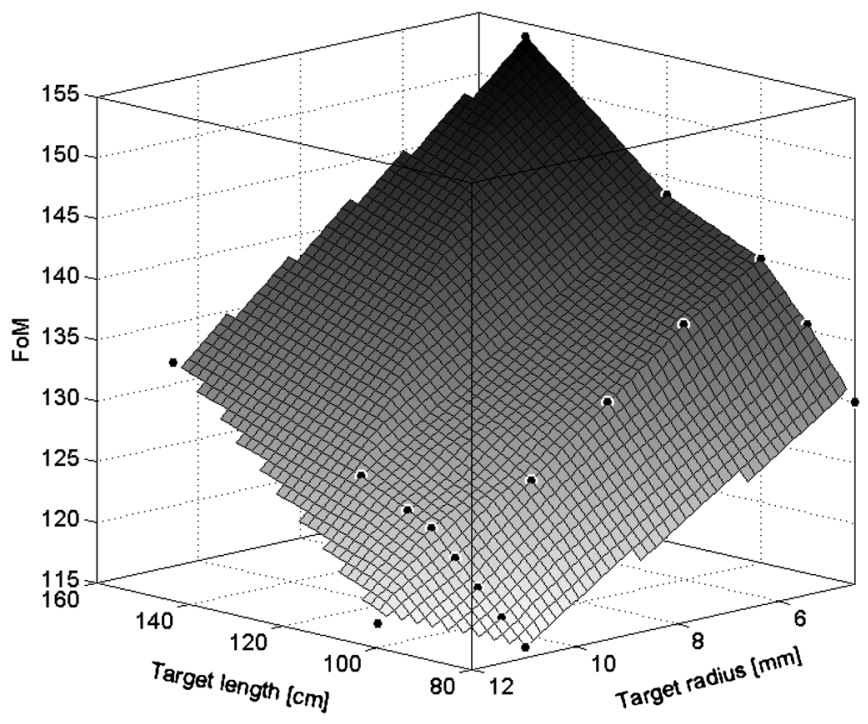

FIG. 7. FOM as a function of target radius and length with target radius $=3 \sigma$. 


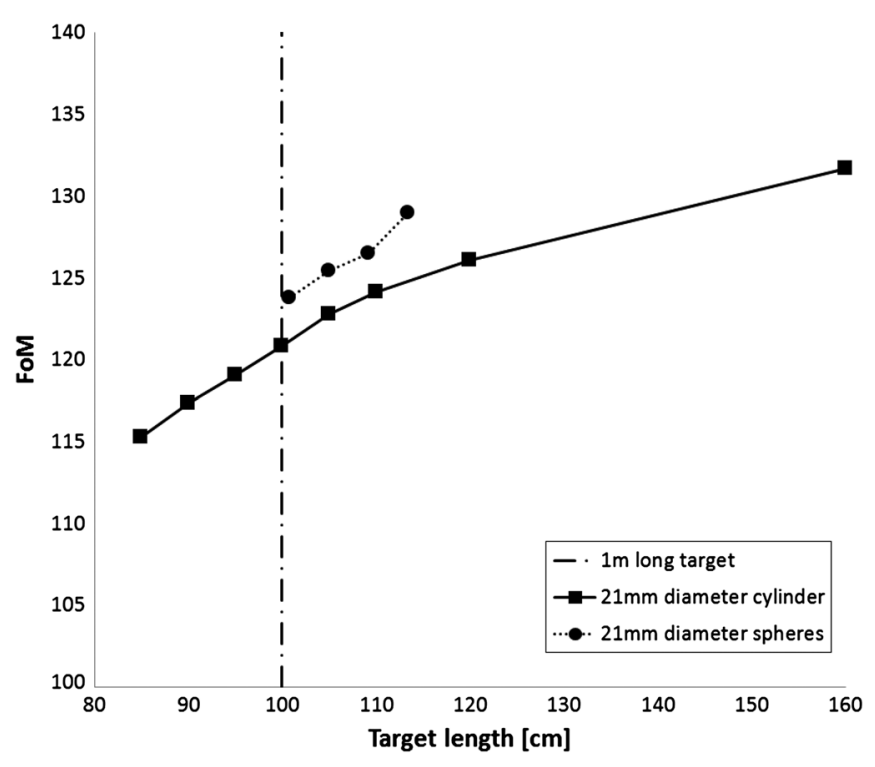

FIG. 8. FOM in spheres and cylinders as a function of target length.

pressure drop from inlet to outlet of the target is predicted to be 1.4 bar. Figure 9 shows the predicted velocity w (in the direction of the beam) in both the annular inlet channel and through the target section. Figure 9 also shows a contour plot of temperature on a plane through the target. It shows the helium temperature increasing along its path through the target and also the temperature at the center of the spheres. The maximum beryllium temperature of $197^{\circ} \mathrm{C}$ predicted by this steady state model occurs near the peak energy deposition region.

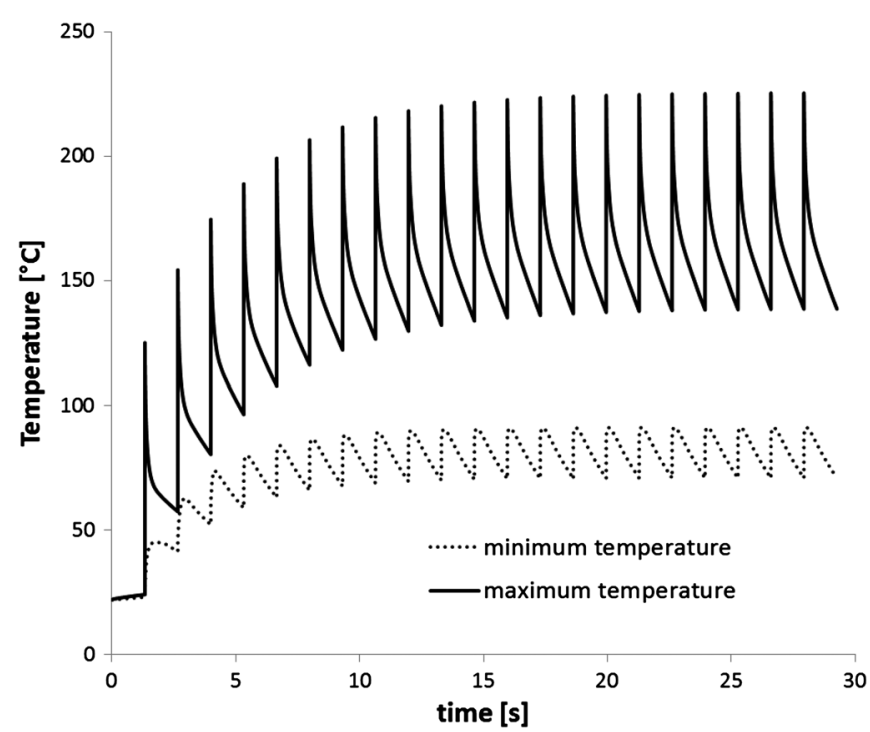

FIG. 10. Transient thermal simulation of minimum and maximum beryllium temperature following 21 beam pulses.

In addition to this steady state simulation which is based on a time averaged heat load, a transient simulation was also carried out. Figure 10 shows the predicted maximum temperature of the beryllium as a result of the pulsed beam heating. A temperature jump per pulse in excess of $100{ }^{\circ} \mathrm{C}$ is predicted with the maximum beryllium temperature of $225^{\circ} \mathrm{C}$ being reached after around ten beam pulses. Beryllium should maintain adequate strength characteristics at this temperature [10]. These parameters would be optimized in a detailed design, however this example

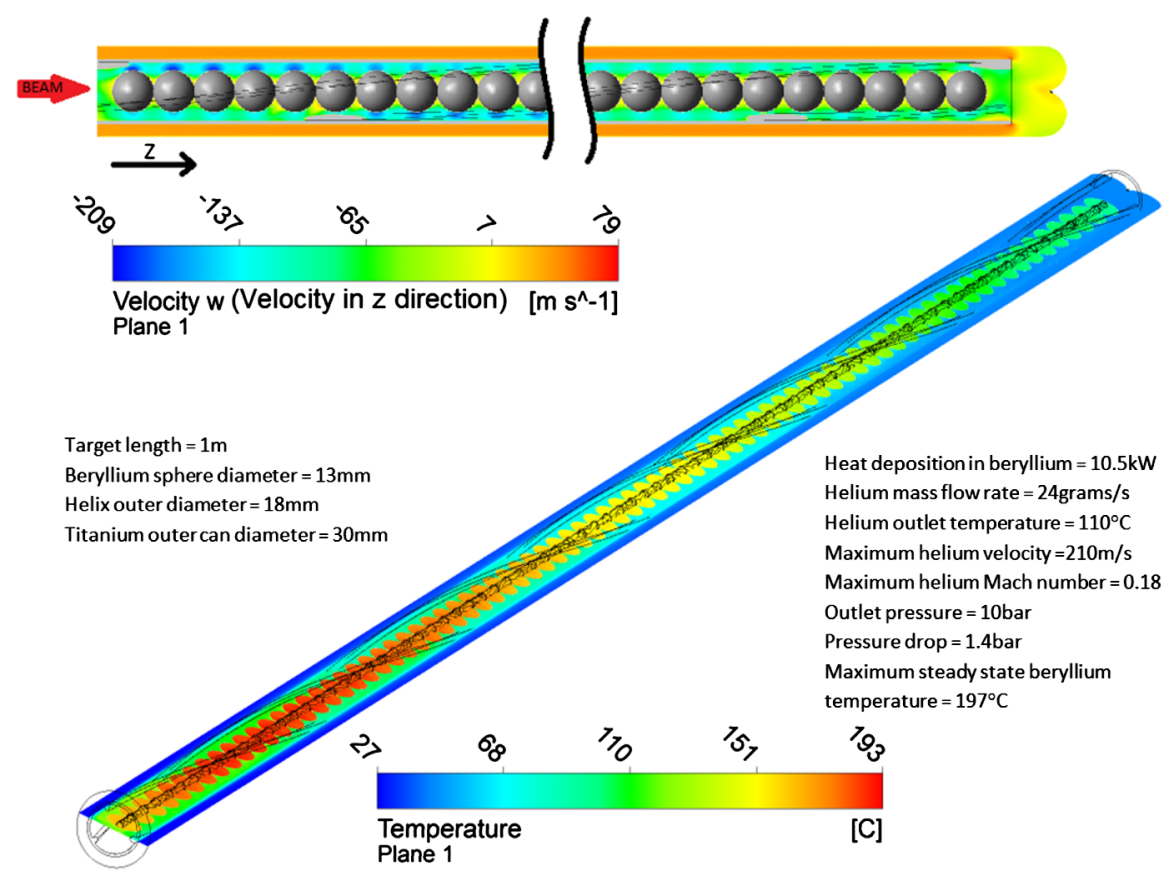

FIG. 9. Contour plots from steady state CFD simulations of a beryllium sphere target showing helium velocity and beryllium temperature. 
highlights a potential beryllium target solution for $2 \mathrm{MW}$ plus super beam facilities.

\section{CONCLUSIONS}

Super beam facilities such as LNBF are being proposed with beam powers in excess of $2 \mathrm{MW}$ with short beam pulses of the order of a few $\mu \mathrm{s}$. These beam parameters result in a significant inertial response in the target material. The magnitude of stress is shown to depend on the characteristic size of the target (as well as on the specific beam parameters). Operational thermal and dynamic stresses in a beryllium target were found to be significantly lower in an array of spheres than in a solid cylindrical target. Lower operational stresses are likely to result in better reliability and lifetime especially in view of the cyclic nature of the thermal loading. A segmented target also offers increased dimensional stability in the case of a nonperfectly centered beam. Generic analysis of the pion yield in the form of a figure of merit shows that a segmented target offers a comparable physics performance to a cylinder of equal radius.

\section{ACKNOWLEDGMENTS}

We would like to acknowledge Fermilab for funding this research programme.
[1] T. Akiri et al., The 2010 interim report of the long-baseline neutrino experiment collaboration physics working groups, arXiv: 1110.6249.

[2] T. R. Edgecock et al., High intensity neutrino oscillation facilities in Europe, Phys. Rev. ST Accel. Beams 16, 021002 (2013).

[3] E. Baussan et al., Neutrino super beam based on a superconducting proton linac, Phys. Rev. ST Accel. Beams 17, 031001 (2014).

[4] K. Abe et al., The T2K experiment, Nucl. Instrum. Methods Phys. Res., Sect. A 659, 106 (2011).

[5] R. Acquafredda et al., First events from the CNGS neutrino beam, New J. Phys. 8, 303 (2006).

[6] J. Strait and S. Childress, Long baseline neutrino beams at Fermilab, arXiv:1304.4899.

[7] O. Caretta, T. Davenne, P. Loveridge, and C. J. Densham, Engineering considerations on targets for a neutrino factory and muon collider, J. Nucl. Mater. 433, 538 (2013).

[8] A. Ferrari, P. R. Sala, A. Fasso, and J. Ranft, FLUKA: A Multi-Particle Transport Code (Stanford University, Stanford, 2005).

[9] P. Sievers, P. Pugnat, and A. Helium, Gas cooled stationary granular target, J. Phys. G 29, 1 (2003).

[10] J. W. Davis and P. D. Smith, ITER material properties handbook, Nucl. Mater. Manage. 233-237, 1593 (1996). 